\title{
Condorcet winners on median spaces
}

\author{
Berno Buechel
}

Received: 18 April 2012 / Accepted: 8 May 2013 / Published online: 30 May 2013

(C) Springer-Verlag Berlin Heidelberg 2013

\begin{abstract}
We characterize the outcome of majority voting for single-peaked preferences on median spaces. This large class of preferences covers a variety of multidimensional policy spaces including products of lines (e.g. grids), trees, and hypercubes. Our main result is the following: If a Condorcet winner (i.e. a winner in pairwise majority voting) exists, then it coincides with the appropriately defined median ("the median voter"). This result generalizes previous findings which are either restricted to a one-dimensional policy space or to the assumption that any two voters with the same preference peak must have identical preferences. The result applies to models of spatial competition between two political candidates. A bridge to the graph-theoretic literature is built.
\end{abstract}

\section{Introduction}

Majority voting is one of the predominant voting methods. Accordingly, many results contribute to our understanding of the properties and limitations of this voting rule. A groundbreaking contribution on this topic is due to Duncan Black who shows the following:

If preferences are single-peaked (on a line), then the median voter's favorite alternative wins in majority voting against any other alternative, i.e. it is a Condorcet winner (Black 1948).

This result is often referred to as the median voter theorem (e.g. Congleton 2002). It is not only a statement about the existence of a Condorcet winner, but also a

\footnotetext{
B. Buechel ( $\square)$

Department of Economics, University of Hamburg, Von-Melle-Park 5, 20146 Hamburg, Germany e-mail: berno.buechel@uni-hamburg.de
} 
characterization of it: the Condorcet winner coincides with the preference peak of the median voter.

Since the domain of single-peaked preferences on a line is quite narrow, it is natural to ask to which extent this assumption can be relaxed. This paper is devoted to address this question.

From the literature it is known that the existence of a Condorcet winner can be extended to single-peaked preferences on tree graphs (Demange 1982). For the characterization of Condorcet winners there are contributions in the field of location theory: for a special class of preferences, Wendell and McKelvey (1981) show that on tree graphs Condorcet winners coincide with the appropriately defined median; Bandelt and Barthélémy (1984) extend this result to so-called cube-free median graphs. Those results apply to interesting multi-dimensional policy spaces. However, they assume that any two voters with the same preference peak have identical preferences. Nehring and Puppe (2007b) have characterized the class of generalized single-peaked preferences on median spaces which subsumes the assumptions of the aforementioned literature as special cases. In this paper we study the outcome of majority voting for this large class of preferences. While the existence of a Condorcet Winner cannot be guaranteed, the characterization given by Black (1948) extends: If there is a Condorcet winner, then it coincides the appropriately defined median ("the median voter").

In their seminal contribution Nehring and Puppe (2007b) discuss single-peaked preferences on median spaces and show that this domain allows for preference aggregation that is neither manipulable nor dictatorial. While, their arguments involve majority voting on (political) issues, they remain silent about majority voting on the alternatives (and thus on Condorcet winners). By using the framework and tools of Nehring and Puppe (2007b) we can directly derive our main result, which is a characterization of the Condorcet winners. Single-peaked preferences have a long tradition in the literature such that their properties and applications are well established (cf. Black 1948; Moulin 1980, 1984; Demange 1982; Ballester and Haeringer 2011).

Independently of this work Demange (2011) has developed some ideas quite similar to the ones presented here. She introduces a notably different set-up, in which a median graph represents the relation of the different preference orderings in a profile. Within this set-up a strong conclusion can be drawn: for strict preferences, the median preference ordering represents the majority relation. While the framework of Demange (2011) is very flexible in principle, it becomes restrictive when one graph is fixed. For example, consider a tree graph with $k$ nodes as a model of a policy space. Within this model there are only $k$ feasible preferences orderings. This is in stark contrast with single-peaked preferences, for which there are at least $2^{k-1}$ strict preference orderings on the same policy space.

To demonstrate the implications of our main result we consider a model of political competition that follows the tradition of Hotelling (1929) and Downs (1957). The policy space in our model is a median space. Thus, it contains the classic example of a line and the well-studied examples of trees as special cases, but also covers other examples, e.g. grids. Moreover, with the assumption of single-peaked preferences, the model does not require that preferences of two voters with the same peak are identical - a ubiquitous feature of other models on spatial competition (see Eiselt and Laporte (1989) for a survey of such models). Based on our characterization of 
the Condorcet winner, the following result is derived: if there is a Nash equilibrium between two Downsian (vote-maximizing) candidates, then it must be such that both candidates choose the appropriately defined median. Moreover, we show that this result is not restricted to Downsian candidates. Assuming that politicians are reformists, i.e. they care about the winning policy (rather than about being the winner), this result still holds if the preferences of the two candidates are "sufficiently heterogeneous."

We will proceed as follows: Section 2 introduces the set-up. Section 3 states the main result. Section 4 presents an application of the result. Section 5 embeds the result into the graph-theoretic literature. Section 6 concludes.

\section{Set-up}

Let $X=\{a, b, c, \ldots\}$ be a finite set of alternatives (e.g. social states, policies, political positions) of size $|X| \geq 3$. Let $N=\{1,2, \ldots, n\}$ be a finite set of voters. The voters are endowed with (complete and transitive) preferences on the set $X$. Let $\succeq:=\left(\succeq_{i}\right)_{i \in N}$ denote a profile of such preferences where $x \succeq_{i} y\left(\begin{array}{lll}x & \succ_{i} & y\end{array}\right)$ denotes that voter $i$ weakly (strictly) prefers $x$ over $y$. Given a preference profile $\succeq$ a Condorcet winner is an alternative that wins in majority voting against any other alternative. More precisely, $x$ is a Condorcet winner if $\forall y \in X$, it holds that $\#\left\{i \in N \mid x \succ_{i} \quad y\right\} \geq \#\{i \in N \mid$ $\left.y \succ_{i} x\right\} .^{1}$

In order to define the relevant domain of preferences, we define property spaces and generalized single-peakedness following Nehring and Puppe (2007b). A set of basic (binary) properties $\mathcal{H}$ is extensionally defined via the alternatives: $\mathcal{H} \subseteq 2^{X}$, where $H \in \mathcal{H}$ stands for a property possessed by exactly all alternatives $x \in H$. A pair $(X, \mathcal{H})$ is called a property space if three conditions are satisfied: (a) properties $H \in \mathcal{H}$ are non-empty; (b) for each property $H \in \mathcal{H}$, the complement, $H^{c}:=X \backslash H$, is also an element of $\mathcal{H}$; and (c) for each pair of alternatives $x \neq y$ there is a property that separates them in the sense that $x \in H$ and $y \notin H$. A pair $\left(H, H^{c}\right)$ is an issue.

A natural relation for a property space is to say that $y$ is between $x$ and $z$ if it shares all of their common properties.

Definition 1 (Betweenness) Let $(X, \mathcal{H})$ be a property space. Define $T_{\mathcal{H}}(\subset X \times X \times X)$ as follows: $\forall x, y, z \in X$

$$
(x, y, z) \in T_{\mathcal{H}} \Longleftrightarrow[\forall H \in \mathcal{H}:\{x, z\} \subseteq H \Rightarrow y \in H]
$$

By definition $(x, x, y) \in T_{\mathcal{H}}$ for any $x, y$. We will consider a special class of property spaces.

Definition 2 (Median Space) A property space $(X, \mathcal{H})$ is a median space if for all $x, y, z \in X$, there exists a unique alternative $m \in X$ such that

$$
\{(x, m, y),(x, m, z),(y, m, z)\} \subseteq T_{\mathcal{H}} .
$$

\footnotetext{
1 Sometimes slightly weaker or slightly stronger notions are considered (cf., e.g. Bandelt 1985).
} 
Median spaces have several desirable properties (cf. Van de Vel 1993), some of which we are going to exploit. ${ }^{2}$ A segment is the set of alternatives between two alternatives: $[x, y]:=\left\{z \in X: \quad(x, z, y) \in T_{\mathcal{H}}\right\}$. Two alternatives $x \neq y$ are neighbors if $[x, y]=\{x, y\}$. Let $N(x)$ denote the set of neighbors of alternative $x$. In a median space any two alternatives $x, y$ are connected via a sequence of neighbors.

The central assumption on preferences will be that they are single-peaked with respect to a given median space.

Definition 3 (Single-peakedness) A profile of preferences $\succeq$ (on $X)$ is single-peaked on the property space $(X, \mathcal{H})$ if any voter's preferences are single-peaked w.r.t. to the betweenness relation $T_{\mathcal{H}}$. That is: for each $i \in N$, there exists $x_{i}^{*} \in X$ such that

$$
\forall y \neq z \in X, \quad\left(x_{i}^{*}, y, z\right) \in T_{\mathcal{H}} \Longrightarrow y \succ_{i} z .
$$

For a characterization and an excellent discussion of single-peaked preferences (on median spaces) we refer the reader, again, to Nehring and Puppe (2007b).

\section{Characterization of the Condorcet winner}

For single-peaked preferences on a line, the "median voter," is the alternative for which it holds that neither to the "left" nor to the "right" there are more than $50 \%$ of the voters' preference peaks. Similarly, we define the median alternative of a property space. ${ }^{3}$ For a profile $\succeq$ of single-peaked preferences on $(X, \mathcal{H})$ and a property $H \in \mathcal{H}$, let the property weight $w(H):=\#\left\{i \in N \mid x_{i}^{*} \in H\right\}$, i.e. the number of voters whose peak satisfies property $H$. Observe that for each issue, we have $w(H)+w\left(H^{c}\right)=n$. The set of median alternatives is then defined as the alternatives that only possess "majority properties," i.e.

$$
\cap_{H \in \mathcal{H}: w(H) \geq \frac{n}{2}} H .
$$

If a profile of preferences is single-peaked on a median space, then a median alternative always exists (Nehring and Puppe 2007b). To ease the exposition, let us assume that the profile $\succeq$ is non-degenerate on $(X, \mathcal{H})$, i.e. there is no property $H$ such that $w(H)=\frac{n}{2}=w\left(H^{c}\right)$, e.g. by assuming that $n$ is odd. Then the median alternative is unique.

The following example illustrates the main definitions and shows that the median alternative need not be a Condorcet winner.

Example 1 Let $X=\{a, b, c, d\}$ and $N=\{1,2,3\}$ with the following strict preference orders $\succeq_{1}=(a, b, c, d), \succeq_{2}=(b, d, a, c), \succeq_{3}=(c, d, a, b)$. This profile of preferences is single-peaked on the property space $\mathcal{H}=\{$ Down $=\{a, b\}, U p=$

\footnotetext{
${ }^{2}$ In particular, for any median space there is a graph $(X, E)$ that represents it in the sense that $(x, y, z) \in T_{\mathcal{H}}$ if and only if $y$ lies on a shortest path between $x$ and $z$.

3 We refer to the median alternative, not the median voter, because in this framework there need not be any voter with preference peak equal to median alternative.
} 
Fig. 1 A median space with four alternatives. If there is one preference peak on each alternative except $d$, then $a$ is the median alternative

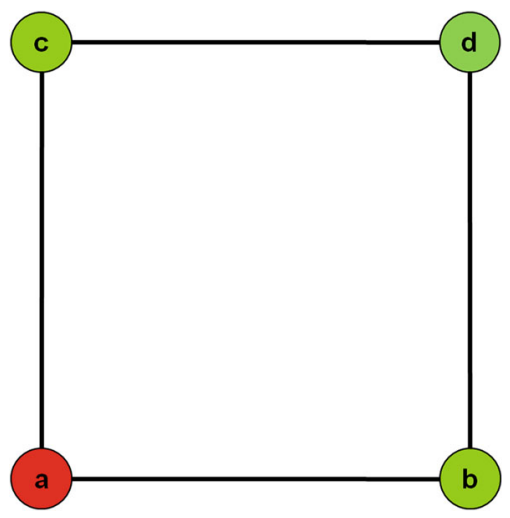

$\{c, d\}$, Left $=\{a, c\}$, Right $=\{b, d\}\}$ (see Fig. 1). Moreover, this is a median space (since for any triple of alternatives one of the alternatives is between the two others and hence between any pair in the triple). The property weights are $w($ Down $)=2, w(U p)=1, w($ Left $)=2$, and $w($ Right $)=1$. The median alternative is thus Left $\cap$ Down $=a$. We observe that alternative $a$ is not a Condorcet winner because $d$ would defeat $a$ by two votes over one. ${ }^{4}$ Note that in this example no Condorcet winner exists, as $b$ and $c$ are defeated by $a$; and $d$ is defeated by $b$ and $c$.

Example 1 shows that the classic characterization of the Condorcet winner does not extend to median spaces in the sense that the median alternative need not be a Condorcet winner. However, since in Example 1 no Condorcet winner exists, one might still conjecture that the median alternative characterizes the Condorcet winner in case they both exist. Prop. 1 shows that this is, indeed, the case on median spaces.

Proposition 1 (Characterization) Let $\succeq$ be single-peaked and non-degenerate on a median space $(X, \mathcal{H})$. If there exists a Condorcet winner, then it coincides with the median alternative.

Proof Let $\succeq$ be as above and let $q$ designate the median alternative. Recall that for any $H \in \mathcal{H}$, if $w(H)>\frac{n}{2}$, then $q \in H$. Take an arbitrary $x \in X \backslash\{q\}$. We will show that $x$ cannot be a Condorcet winner.

1. $[x, q] \cap N(x) \neq\{\varnothing\}$ because a median space is connected via pairs of neighbors.

2. Let $y \in[x, q] \cap N(x)$. In median spaces neighbors differ in exactly one issue (Lemma B.3 in Nehring and Puppe (2007b)). Let $\left(H, H^{c}\right)$ be the issue that separates $x$ from $y$, with $x \in H$, and $y \in H^{c}$.

3. $\forall z \in H^{c}$, we have $(z, y, x) \in T_{\mathcal{H}}$. This is because any property that is shared by $z$ and $x$ is also shared by $y$ (since $x$ and $y$ only differ with respect to $\left(H, H^{c}\right)$ ). Thus, single-peakedness implies $\forall i \in N$ s.t. $x_{i}^{*} \in H^{c}$, that $y \succ_{i} x$.

\footnotetext{
4 By definition $a$ would win a majority voting on the issues (Nehring and Puppe 2007b), but not majority voting on the alternatives.
} 
4. $q \in H^{c}$ because $(q, y, x) \in T_{\mathcal{H}}$ (by definition of $y$ ). Thus, $w\left(H^{c}\right)>\frac{n}{2}>w(H)$ (by definition of $q$ ). Thus, $y$ defeats $x$ (in the sense that strictly more voters vote for $y$ against $x$ ).

5. We conclude that $x(\neq q)$ cannot be a Condorcet winner. If there is a Condorcet winner, then it must be $q$.

We have shown that any alternative that is not the median is defeated in majority voting by some of its neighbors. For that purpose, we use a neighbor that is between the alternative and the median alternative. The condition that $(X, \mathcal{H})$ is a median space is necessary for this result. If preferences are single-peaked on a non-median space, then a median alternative (as defined here) need not exist and even if it does, the median alternative and the Condorcet winner do not coincide, in general. ${ }^{5}$ To illustrate the importance of this result, we now consider its implications for a model of political competition.

\section{Application to political competition}

Consider two political candidates $A$ and $B$ who are able to propose a political position. Formally, a strategy for each candidate $k \in\{A, B\}$ is to pick an alternative $s^{k} \in$ $S^{k}=X$. The strategy space is $S=X^{2}$. Given a strategy profile $s \in S$, each voter's preferences over the alternatives $X$ induce preferences over the candidates $A, B$. Fixing a preference profile $\succeq$ let the outcome rule $\phi: S \rightarrow \mathbb{R}^{2}$ keep track of how many voters prefer $s^{A}$ over $s^{B}$ and vice versa, where indifferent voters are counted with weight $\frac{1}{2}$ in both entries $\phi^{A}(s)$ and $\phi^{B}(s)$ of the vector $\phi(s)$. Let $\rho(s)$ be the winning alternative, i.e. the strategy of the candidate with a majority of voters-more precisely, $\rho(s)=s^{A}$ if and only if $\phi^{A}(s) \geq \phi^{B}(s){ }^{6}$

For the payoffs of the candidates we consider two different assumptions. If the candidates want to maximize their number of votes, i.e.

$$
\pi^{k}(s):=\Pi^{k}\left(\phi^{k}(s)\right)
$$

for some increasing function $\Pi^{k}$, we will call them Downsian. ${ }^{7} \Pi^{k}$ need not be strictly increasing; however, we assume that $\Pi^{k}\left(\frac{n}{2}-\varepsilon\right)<\Pi^{k}\left(\frac{n}{2}\right)<\Pi^{k}\left(\frac{n}{2}+\varepsilon\right)$ for any $\varepsilon>0$. Alternatively, let a reformist be a candidate who is not concerned about winning the election, but about $\rho(s)$, the political position that wins the election, (because this determines the policy that is finally implemented). So, we here assume that the

\footnotetext{
5 The latter point will be illustrated in Example 2.

${ }^{6}$ For convenience, we let $A$ be the winning candidate in case of a tie. If $s^{A}=s^{B}$, then this convention does not matter.

7 This modeling approach was made prominent by Downs (1957) who writes: "political parties in a democracy formulate policy strictly as a means of gaining votes. They do not seek to gain office in order to carry out certain preconceived policies or to serve any particular interest groups; rather they formulate policies and serve interest groups in order to gain office." The Downsian approach has been critized by Roemer (2001), among others.
} 
candidates themselves are endowed with preferences such as voters are. ${ }^{8}$ Let $\succeq^{k}$ stand for the preferences of candidate $k \in\{A, B\}$ on the set $X$. A reformist $k$ ranks strategy profiles in the following way: $s$ is preferred to $s^{\prime}$ if and only if $\rho(s) \succeq^{k}$ $\rho\left(s^{\prime}\right)$. Both assumptions constitute a normal-form game: one for Downsian candidates $\Gamma^{\succeq}=\left(\{A, B\}, S,\left(\pi^{A}, \pi^{B}\right)\right)$ and one for reformists $\tilde{\Gamma}^{\succeq}=\left(\{A, B\}, S,\left(\succeq^{A}, \succeq^{B}\right)\right)$. The games are set-up simultaneously, while sequential moves would not change the results.

\subsection{Downsian candidates}

It follows from the definitions that in a game of Downsian candidates determining the winner for some strategy profile coincides with the outcome of majority voting on the two alternatives $s^{A}$ and $s^{B}$. Thus, a strategy profile is a Nash equilibrium if and only if strategies are Condorcet winners. This leads to the following corollary of Proposition 1, where $N E(\Gamma)$ denotes the set of Nash equilibria of a game $\Gamma$.

Corollary 1 (Downsian candidates) Let $\succeq$ be single-peaked and non-degenerate on a median space $(X, \mathcal{H})$ with median $q$. Let $\Gamma^{\succeq}$ be a game of two Downsian candidates. Then

$$
\left(s^{A}, s^{B}\right) \in N E\left(\Gamma^{\succeq}\right) \Longrightarrow s^{A}=s^{B}=q .
$$

If an equilibrium exists, then it is such that both candidates choose the median alternative. This result extends the classic observation of minimal differentiation at the median (Hotelling 1929) to single-peaked preferences on median spaces. It might seem that this result is fully driven by the assumption of Downsian candidates. However, the Nash equilibria for reformists have similar properties, as we will show next.

\subsection{Reformists}

Consider two reformists $A, B$ who have single-peaked preferences on a property space $\mathcal{H}$ with median alternative $q$. We say that preferences of the reformists are sufficiently heterogeneous if it holds that $\left(x_{*}^{A}, q, x_{*}^{B}\right) \in T_{\mathcal{H}}$. This is interpreted as having the preference peak located in a different part of the property space. ${ }^{9}$ Another interpretation is that the preference peaks of the candidates do not share a property that is only supported by a minority of voters. Indeed, by definition of betweenness $\left(x_{*}^{A}, q, x_{*}^{B}\right) \in T_{\mathcal{H}}$ if and only if it holds that $\left[\left\{x_{*}^{A}, x_{*}^{B}\right\} \subseteq H \Rightarrow q \in H\right]$, which implies $w(H) \geq \frac{n}{2}$. The following result shows that for sufficiently heterogeneous reformists, the outcome of political competition is the median alternative.

Proposition 2 (Reformists) Suppose $\succeq$ is single-peaked and non-degenerate on a median space $(X, \mathcal{H})$ and there exists a Condorcet winner. Let $q$ designate the

\footnotetext{
8 This idea is similar to the "citizen-candidates" introduced in Osborne and Slivinski (1996).

9 One could argue that if the opponent has very similar preferences, a reformist's incentive to run for election might just not be strong enough.
} 
median alternative (and the Condorcet winner). Let $\tilde{\Gamma}^{\succeq}$ be a game of two reformists, where $\left(\succeq^{A}, \succeq^{B}\right)$ is single-peaked on $(X, \mathcal{H})$. If the candidates' preferences are sufficiently heterogeneous, i.e. $\left(x_{*}^{A}, q, x_{*}^{B}\right) \in T_{\mathcal{H}}$, then $(i)(q, q) \in N E\left(\tilde{\Gamma}^{\succeq}\right)$ and (ii) $s^{*} \in N E\left(\tilde{\Gamma}^{\succeq}\right) \Rightarrow \rho\left(s^{*}\right)=q$.

Proof (i) Since $q$ is a Condorcet winner (by Proposition 1), $\rho\left(q, s^{k}\right)=q$ for any $s^{k}$ with $k \in\{A, B\}$. Thus, $\nexists s^{k \prime}$ that is an improvement for $k$. Thus, $(q, q) \in N E\left(\tilde{\Gamma}^{\succeq}\right)$.

(ii) We show that if $s$ is such that $\rho(s) \neq q$, then $s \notin N E\left(\tilde{\Gamma}^{\succeq}\right)$.

Let $\rho(s) \neq q$, w.l.o.g. let $\rho(s)=s^{A}$. Let $Y:=\left[x_{*}^{B}, s^{A}\right] \cap\left[q, s^{A}\right]$. Note first that $s^{A} \in Y$.

Case $1 Y=\left\{s^{A}\right\}$. Since $(X, \mathcal{H})$ is a median space, there exists an alternative between the triple $q, s^{A}, x_{*}^{B}$. Thus, $\left(q, s^{A}, x_{*}^{B}\right) \in T_{\mathcal{H}}$. Sufficient heterogeneity means that $\left(x_{*}^{A}, q, x_{*}^{B}\right) \in T_{\mathcal{H}}$. Therefore, $\left(x_{*}^{A}, q, s^{A}\right) \in T_{\mathcal{H}}$.

Single-peakedness then implies that $q \succ^{A} s^{A}=\rho(s)$. However, for (any) $s^{B}$ it holds that $\rho\left(q, s^{B}\right)=q$ because $q$ is a Condorcet winner (by Proposition 1). Therefore, $s^{A^{\prime}}=q$ is an improving deviation for candidate $A$.

Case $2 Y \supsetneqq\left\{s^{A}\right\}$, i.e. $\exists m \in Y$ s.t. $m \neq s^{A}$. $\left[m, s^{A}\right] \cap N\left(s^{A}\right) \neq \varnothing$ because in a median space any two alternatives are connected via a sequence of neighbors. Let $y \in\left[m, s^{A}\right] \cap N\left(s^{A}\right)$. Because $m \in Y$ and $\left(m, y, s^{A}\right) \in T_{\mathcal{H}}$, it holds that $y \in Y$. Thus, $\left(x_{*}^{B}, y, s^{A}\right) \in T_{\mathcal{H}}$, implying that $y \succ^{B} s^{A}=\rho(s)$. Moreover, $s^{A}$ and $y$ differ with respect to exactly one issue-say $y \in H, s^{A} \in H^{c}$ - since this holds true for any pair of neighbors. Therefore, $\forall z \in H$ we have $\left(z, y, s^{A}\right) \in T_{\mathcal{H}}$. Thus, single-peakedness implies for a voter $i, y \succ_{i} s^{A}$ if and only if $x_{i}^{*} \in H$. This implies that $\rho\left(s^{A}, y\right)=y$ because $H^{c}$ is a majority property (indeed, $q \in H$ means that $w(H)>w\left(H^{c}\right)$ by construction of $q$ ). Therefore, $s^{B \prime}=y$ is an improving deviation for candidate $B$.

Proposition 2 establishes that if a Condorcet winner exists and it lies between the two reformists' peaks, then (i) we have a Nash equilibrium with both candidates choosing the median alternative and (ii) in any Nash equilibrium the winning alternative is the median alternative. For the first part (i) the assumption of heterogeneous preferences is not necessary, but it is necessary for the second part. Consider $x_{*}^{A}=x_{*}^{B} \neq q$, which is a special case of a violation of sufficient heterogeneity. Then $s^{A}=s^{B}=$ $x_{*}^{A}$ is also a Nash equilibrium (because this outcome is payoff-maximal for both reformists). Generally, we have a multitude of Nash equilibria with several different winning alternatives if sufficient heterogeneity is violated. The assumption that a Condorcet winner exists is necessary for both part (i) and part (ii) of Proposition 2. Reconsider Example 1 where no Condorcet winner existed. Let $\succeq^{A}=(a, b, c, d)$ and $\succeq^{B}=(b, d, a, c)$. Then $s^{A}=b\left(=x_{*}^{B}\right)$ and $s^{B}=d$ constitute the unique Nash equilibrium with $\rho(s)=b \neq a$, while $a$ is the median alternative.

The two results, Corollary 1 and Proposition 2, show that under weak assumptions the outcome of political competition on a median space is the median alternative. ${ }^{10}$ Let us now compare our results with similar findings.

10 An interesting variation of the two games $\Gamma^{\succeq}$ and $\tilde{\Gamma}^{\succeq}$ is to consider the competition of one Downsian candidate and one reformist. It turns out that, similar to the violation of sufficient heterogeneity between 


\section{Relation to literature}

To clarify the relation of our results to the existing literature, it is helpful to reformulate our model in graph-theoretic terms.

\subsection{Median graphs and Weber points}

Let $G=(X, E)$ be a graph, where the nodes are identified with the alternatives $X$, and $E$ is the set of (undirected, unweighted) edges. We write $x y \in E$ for an edge linking nodes $x \in X$ and $y \in X$. The (geodesic) distance $d(x, y)$ of two nodes $x, y$ is the number of edges in a shortest path between them. ${ }^{11}$ A node $y$ is graphicly between two nodes $x$ and $z$ if $y$ belongs to a shortest path between $x$ and $z$.

Definition 4 (Graphic Betweenness) Let $(X, E)$ be a graph. Define $T_{E}(\subset X \times X \times X)$ as follows: $\forall x, y, z \in X$

$$
(x, y, z) \in T_{E} \Longleftrightarrow d(x, z)=d(x, y)+d(x, z) \text {. }
$$

If a graph $(X, E)$ satisfies a separability condition, then there is a property space $(X, \mathcal{H})$ whose betweenness relation (cf. Definition 1) coincides with the graphic betweenness relation, i.e. $T_{\mathcal{H}}=T_{E}$ (Nehring and Puppe 2007b). On the other hand, for a sizable class of property spaces, which contains all median property spaces, there exists a corresponding graph that represents it in that sense. Thus, for median property spaces there always exists a graph representation in the sense that $(x, y, z) \in T_{\mathcal{H}}$ if and only if $y$ lies on a shortest path between $x$ and $z .^{12}$

We can now reconsider voters $N$ with preferences on the alternatives $X$. The notion of single-peaked preferences on a graph is readily defined by switching the notion of betweenness in Definition 3 to graphic betweenness. Thus, a profile of preferences $\succeq$ is single-peaked on a graph $(X, E)$ if for every voter $i$ there exists a favorite node $x_{i}^{*}$ such that $\forall y \neq z \in X,\left(x_{i}^{*}, y, z\right) \in T_{E}$ implies $y \succ_{i} z$. This means that any alternative $y$ (including $y=x_{i}^{*}$ ) is strictly preferred over another alternative $z$ that lies on a shortest path from $x_{i}^{*}$ to $z .^{13}$

For median graphs, the framework of single-peaked preferences on graphs and the framework of single-peaked preferences on the corresponding property space can

Footnote 10 continued

reformists, both candidates choosing the Condorcet winner is a Nash equilibrium, but generically there are other Nash equilibria with different outcomes.

11 A path from $x$ to $y$ is a sequence of distinct nodes $\left(x_{1}, \ldots, x_{T}\right)$ such that $x_{1}=x, x_{T}=y$, and $x_{t} x_{t+1} \in E$ for all $t \in\{1, \ldots, T-1\}$. We focus on graphs that are connected, i.e. there exists a path between any pair of nodes, and we define $d(x, x)=0$ for any node $x$ and any graph.

12 In particulr, these median graphs satisfy that for any triple of nodes $x, y, z \in X$, there is exactly one node $m$, which is graphicly between each pair of the triple. Median graphs are well-studied objects and contain lines, trees, products of lines (grids), and hypercubes, as special cases (Van de Vel 1993).

13 The restrictiveness of the assumption of single-peakedness depends on the graph structure, e.g. for the line graph we have the standard notion of single-peakedness, while for the complete graph, we obtain the unrestricted domain (Nehring and Puppe 2007b). 
be used interchangeably since $T_{\mathcal{H}}=T_{E}$. Thereby, median alternatives translate into Weber points. For a graph $(X, E)$ and a profile of peaks $\left(x_{i}^{*}\right)_{i \in N}$ a Weber point (or just "median" of the graph) is a node $x$ that minimizes the distances to all peaks, i.e. $\arg \min _{x \in X} \sum_{i \in N} d\left(x, x_{i}^{*}\right)$. We can now neatly embed our results into the graphtheoretic framework and compare them with previous findings.

\subsection{Results for distance-based preferences}

In the literature on location theory, there are contributions to majority voting on graphs (cf. Hansen et al. (1986) for a bridge into this literature). However, virtually all of them narrow their interest to a specific class of preferences, which we call distance-based: A voter is assumed to cast her vote for the candidate that is closest to her. More precisely, preferences $\succeq$ are distance-based on a graph $(X, E)$ if for any voter $i$ there is a peak $x_{i}^{*}$ and it holds that $x \succeq_{i} y$ if and only if $d\left(x_{i}^{*}, x\right) \leq d\left(x_{i}^{*}, y\right)$. A profile of distance-based preferences is a special case of single-peaked preferences on the same graph. ${ }^{14}$

For distance-based preferences a Condorcet winner is a node that is closer to more peaks than any node it is compared with-a so-called plurality point. For this type of Condorcet winners existence and characterization results can be found. Wendell and McKelvey (1981) show that on a tree graph, i.e. connected graphs without circles, Condorcet winners (plurality points) always exist and coincide with the Weber points. Bandelt and Barthélémy (1984) extend this result to median graphs that do not contain three-dimensional hypercubes (what they call "cube-free" median graphs). In that light our characterization of Condorcet winners as median alternatives (Proposition 1) is not surprising (because median alternatives correspond to Weber points). Also Corollary 1 resembles existing statements-in particular, a corresponding result holds for a model of spatial competition on tree graphs (Hansen et al. 1986). However, it is important to note that those former results are only established for the special case of distance-based preferences. The assumption of distance-basedness is very strong for two reasons: (a) it imposes a symmetry requirement for the preference of each voter; (b) it imposes homogeneity of preferences for all voters who share the same peak. The symmetry requirement (a) can be relaxed with the introduction of varying edge-lengths (e.g. Wendell and McKelvey 1981; Bandelt 1985). The homogeneity requirement (b), however, is inherent in the assumption of distance-based preferences: any distance between two alternatives is valued equally by all voters. Thus, for each graph $(X, E)$ there are only $|X|$ possible distance-based preference orderings-one for each node of the graph-while single-peaked preferences are much more general than this.

\subsection{Results for single-peaked preferences}

In graph-theoretic terms, the classic median voter theorem due to Black (1948) shows that for single-peaked preferences on a line graph a Condorcet winner exists and

\footnotetext{
14 Thus, fixing a graph and a distribution of preference peaks, there are many profiles of single-peaked preferences, while only one of them is distance-based.
} 
Fig. 2 Single-peaked preferences on a graph which is not a median graph. Alternative $a$ is the Weber point, while alternative $d$ is the Condorcet winner

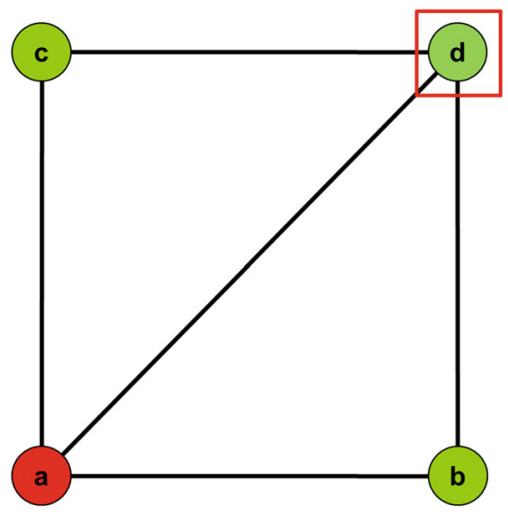

coincides with the Weber point. The guarantee of existence can be extended to singlepeaked preferences on tree graphs (Demange 1982). This is, however, the largest class of graphs for which single-peaked preferences always admit a Condorcet winner.

Proposition 3 (Existence) A graph (X, E) guarantees existence of a Condorcet winner for any profile of single-peaked preferences if and only if it is a tree.

The proof of Proposition 3 is relegated to an appendix. The "if part" is implied by basic properties of trees; the "only if" part applies the idea of Example 1 to all possible cases of graphs that are not trees. Note that the lack of a guarantee for existence does not imply that the existence of a Condorcet winner is unlikely in some probabilistic sense. ${ }^{15}$ Proposition 1 has established that the characterization of Condorcet winners holds for all median spaces. Thus, in a median graph with a non-degenerate distribution of voters, there is only one potential Condorcet winner, the Weber point. We finally show by means of an example that this result does not hold for single-peaked preferences on arbitrary graphs.

Example 2 Let $X=\{a, b, c, d\}$ and $E=\{a b, b d, d c, c a, a d\}$ (see Fig. 2). This is not a median graph, e.g. for the triple $a, b, d$ there is no alternative that is graphicly between each pair of the triple. Let $N=\{1,2,3\}$ be three voters with the following strict preference orders $\succeq_{1}=(a, d, b, c), \succeq_{2}=(b, d, a, c), \succeq_{3}=(c, d, a, b)$. The preferences in Example 2 differ from those in Example 1 only with respect to voter 1. This profile of preferences is single-peaked on the graph $(X, E)$ but it would not be single-peaked on the graph of Example 1, i.e. $(X, E \backslash\{a d\})$.

Alternative $a$ is again the Weber point (since its distance to all peaks is two, while this number is three for all other nodes). However, alternative $d$ is the Condorcet winner. Thus, the characterization that any Condorcet winner must be a Weber point does not hold for this graph.

\footnotetext{
15 Median graphs that are not trees might well admit a Condorcet winner for many profiles of single-peaked preferences. For example, a Condorcet Winner did not exist for the profile $\succeq$ given in Example 1 . If we consider a change of preferences of voters 2 and 3 such that $\succeq_{2}^{\prime}=(b, a, d, c), \succeq_{3}^{\prime}=(c, a, d, b)$, i.e. these voters now prefer $a$ over $d$, then the Weber point, alternative $a$, becomes a Condorcet winner. Other nodes can never be Condorcet winners for single-peaked preferences on this graph (by Proposition 1).
} 


\section{Concluding remarks}

This paper has analyzed the conditions under which the outcome of majority voting can be characterized by the median alternative - the statement that is also referred to as the median voter theorem (e.g. Congleton 2002). Classically, it is known to hold for single-peaked preferences on a line (Black 1948). But also, for distance-based preferences on some median graphs, which contain lines, trees, and grids as special cases (Bandelt and Barthélémy 1984). By showing that the result extends to singlepeaked preferences on median spaces (Proposition 1), we generalize these previous findings. This analysis reveals that potentially critical assumptions are not driving the result. The characterization of the Condorcet winner is neither restricted to onedimensional policy spaces (Black 1948); nor to preferences that require homogeneity of voters who share the same preference peak (Bandelt and Barthélémy 1984).

Applying our main result to a model of political competition on median spaces leads to the following classic observation: In equilibrium, two political candidates choose the median alternative (Corollary 1 and Proposition 2). However, it should be kept in mind that Nash equilibria need not exist in this general set-up (cf. Example 1). Their existence is guaranteed only for tree graphs (Proposition 3), i.e. one-dimensional median spaces according to the classification of Nehring and Puppe (2007a).

The reformulation of single-peaked preferences on property spaces to single-peaked preferences on graphs builds a bridge into the literature of location theory and of spatial competition. Models of competitive location choices on graphs, cf. Eiselt and Laporte

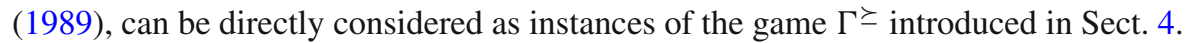
It is crucial to note that in this literature the profile of preferences $\succeq$ is distance-based. This is a natural assumption if the graph represents geographic locations, since long (short) distances imply high (low) transportation costs for any agent. If the graph represents a policy space, then this assumption is highly questionable: two voters with the same moderate political position might well differ in their ordering of left-wing and right-wing extremists. However, our results show that the two player results on median spaces are robust in the sense that they do not rely on the assumption of distance-basedness.

Results for more than two players are also established for distance-based preferences only (e.g. Eaton and Lipsey 1975; Eiselt and Laporte 1993). Thus, an interesting open problem is the characterization of Nash equilibria between several players when voters' preferences are single-peaked on a graph.

Acknowledgments I thank Veronica Block, Dinko Dimitrov, Jan-Philip Gamp, Anke Gerber, ClausJochen Haake, Tim Hellmann, Dominik Karos, Gerd Muehlheusser, Clemens Puppe, Nils Roehl, Walter Trockel, and the participants of the "Public Economic Theory (PET 10)" Conference in Istanbul and the "SING 9" Workshop in Budapest for helpful discussions and comments. Moreover, this paper has benefited from the suggestions of two anonymous referees.

\section{Appendix: Proof of Proposition 3}

Proposition 3 (Existence) A graph (X,E) guarantees existence of a Condorcet winner for any profile of single-peaked preferences if and only if it is a tree. 
Proof We have to prove two directions.

IF Suppose $\succeq$ is a profile of single-peaked preferences on a tree graph $(X, E)$. Let $q$ be a Weber point, i.e. $q \in \arg \min _{x \in X} \sum_{i \in N} d\left(x, x_{i}^{*}\right) .{ }^{16}$ Consider any alternative $x \neq q$. We will show that $x$ cannot be preferred over $q$ by a majority of voters.

Since $(X, E)$ is a tree graph, there is a unique path between $q$ and $x$. Let $N(q):=\{y \in X \mid q y \in E\}$, i.e. the set of graphic neighbors of node $q$. Let $y$ be the neighbor of $q$ that lies graphicly between $q$ and $x$, i.e. $y \in N(q)$ such that $(x, y, q) \in T_{E}$. Since trees are connected without circles, we have for any node $z$, either $d(z, y)<d(z, q)$ or $d(z, q)<d(z, y)$. A well-known result on tree graphs is that for a profile of peaks $\left(x_{i}^{*}\right)_{i \in N}$ a Weber point $q$

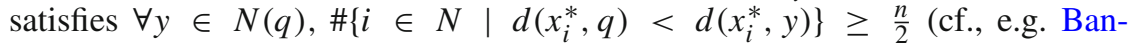
delt and Barthélémy 1984). Thus, for at least $\frac{n}{2}$ voters we have $d\left(x_{i}^{*}, q\right)<$ $d\left(x_{i}^{*}, y\right)$, which means that $\left(x_{i}^{*}, q, x\right) \in T_{E}$ such that single-peakedness implies $q \succ_{i} x$.

ONLY IF For any graph that is not a tree, we will provide a single-peaked preference profile of three voters such that there is no Condorcet winner. Trees are connected and cyclic. Recall that a circle (or cycle) $\mathcal{K}$ is a sequence of $K(\geq 3)$ distinct nodes $\left(x_{1}, \ldots, x_{K}\right)$ such that $x_{k} x_{k+1} \in E$ for all $k \in$ $\{1, \ldots, K-1\}$ and $x_{K} x_{1} \in E$. We first consider unconnected graphs (case 1), then connected graphs where the smallest circle is of odd length (case 2), and finally connected graphs where the smallest circle is of even length (case 3 ).

Case $1(X, E)$ is unconnected. Then there are two nodes, say $a$ and $b$, which are not connected by any path. Let $c$ be some other node. Note that none of the three nodes $a, b, c$ is graphicly between the two others. Denote $R:=X \backslash$ $\{a, b, c\}$. Consider three voters $N$ with the following strict preference orderings: $\left(a, b, c, \sigma_{1}(R)\right),\left(b, c, a, \sigma_{2}(R)\right)$, and $\left(c, a, b, \sigma_{3}(R)\right)$, where $\sigma_{i}$ are orders on $R$ which do not violate single-peakedness. This profile of preferences is single-peaked on $(X, E)$. In majority voting any alternative in $R$ is strictly defeated, while among the three alternatives $a, b, c$, we have the classic Condorcet cycle.

Case $2(X, E)$ is connected and the smallest circle is of odd length $K$. If $K=3$, denote a smallest circle by $\mathcal{K}=(a, b, c)$ and let $R:=X \backslash\{a, b, c\}$. Consider three voters with preferences on $a, b, c$ exactly as in case 1 and the preferences on the rest $R$ are added in some single-peaked order $\sigma_{i}$. This profile of preferences is single-peaked on $(X, E)$. There is no Condorcet winner such as in case 1 .

Now, let $K \geq 5$. Let us consider one smallest circle $\mathcal{K}$ and label it as follows: $\mathcal{K}=$ $\left(a, b_{1}, b_{2}, \ldots, b_{\frac{K-1}{2}}, d, c_{\frac{K-1}{2}-1}, c_{\frac{K-1}{2}-2}, \ldots, c_{1}\right)$ such as illustrated in Fig. 3 panel (a). Let $Z$ denote the set of nodes on the circle. Now, consider three voters with the following strict preference orderings:

$\overline{16}$ A Weber point certainly exists but need not be unique. 

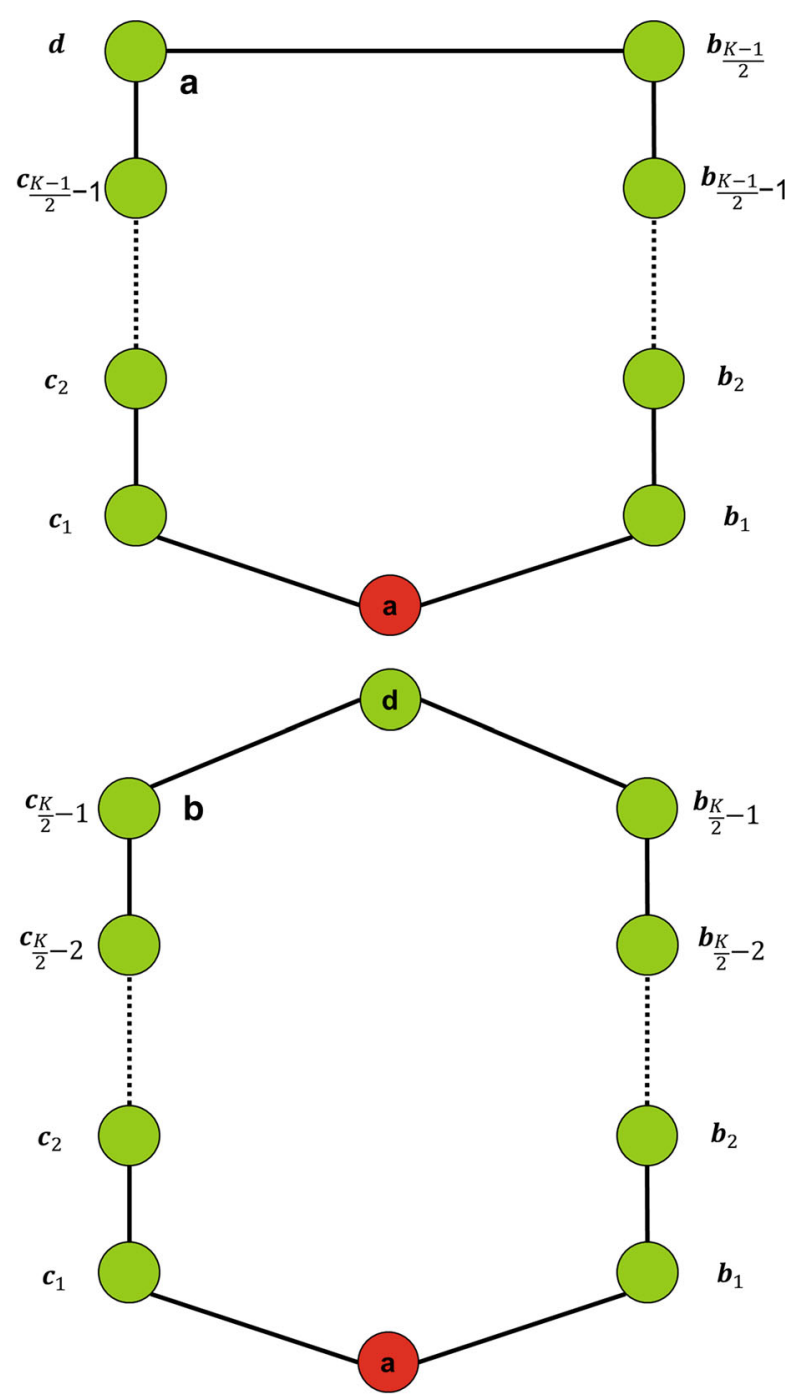

Fig. 3 A circle of length $K$, where $K$ is odd in panel (a) and $K$ is even in panel (b)

$$
\begin{aligned}
& \succeq_{1}=\left(a, b_{1}, c_{1}, b_{2}, c_{2}, \ldots, b_{\frac{K-1}{2}-1}, c_{\frac{K-1}{2}-1}, b_{\frac{K-1}{2}}, d, \sigma_{1}(X \backslash Z)\right) \\
& \succeq_{2}=\left(b_{1}, b_{2}, \ldots, b_{\frac{K-1}{2}}, d, a, c_{1}, c_{2}, \ldots, c_{\frac{K-1}{2}-1}, \sigma_{2}(X \backslash Z)\right) \\
& \succeq_{3}=\left(c_{1}, c_{2}, \ldots, c_{\frac{K-1}{2}-1}, d, a, b_{1}, b_{2}, \ldots, b_{\frac{K-1}{2}}, \sigma_{3}(X \backslash Z)\right),
\end{aligned}
$$

where the alternatives in $X \backslash Z$ are listed in some single-peaked order $\sigma_{i}$. Since $\mathcal{K}$ is a smallest circle, there are no paths between members of the circle which are shorter than the shortest path on the circle. Thus, there is no node $y \in X \backslash Z$ such that $(x, y, z) \in T_{E}$ for some $x, z \in Z$. Therefore, the profile $\succeq$ is single-peaked on $(X, H)$. In majority voting alternative $a$ defeats any alternative except $d$. $b_{1}$ defeats $d$ and, finally, $d$ defeats $a$. 
Case $3(X, E)$ is connected and the smallest circle is of even length $K$. Let us consider one smallest circle $\mathcal{K}$ and label it as follows: $\mathcal{K}=\left(a, b_{1}, b_{2}, \ldots, b_{\frac{K}{2}-1}, d\right.$, $\left.c_{\frac{K}{2}-1}, c_{\frac{K}{2}-2}, \ldots, c_{1}\right)$ such as illustrated in Fig. 3 panel (b). Let $Z$ denote the set of nodes on the circle. Any pair $x, y \in Z$ has a shortest path using only nodes of the circle. However, there might be additional shortest paths for pairs at the opposite sides of the circle, i.e. when $d(x, y)=\frac{K}{2} \cdot{ }^{17}$ Let $Y$ denote the set of all nodes that belong to such a path but not to the circle, i.e. $Y:=\left\{y \in X \backslash Z \mid \exists x, z \in Z\right.$ with $\left.(x, y, z) \in T_{E}\right\}$, and let $R:=X \backslash(Z \cup Y)$. Now, consider three voters with the following strict preference orderings:

$$
\begin{aligned}
& \succeq_{1}=\left(a, b_{1}, c_{1}, b_{2}, c_{2}, \ldots, b_{\frac{K}{2}-1}, c_{\frac{K}{2}-1}, \sigma_{1}(Y), d, \tilde{\sigma}_{1}(R)\right) \\
& \succeq_{2}=\left(b_{1}, b_{2}, \ldots, b_{\frac{K}{2}-1}, d, a, c_{1}, c_{2}, \ldots, c_{\frac{K}{2}-2}, \sigma_{2}(Y), c_{\frac{K}{2}-1}, \tilde{\sigma}_{2}(R)\right) \\
& \succeq_{3}=\left(c_{1}, c_{2}, \ldots, c_{\frac{K}{2}-1}, d, a, b_{1}, b_{2}, \ldots, b_{\frac{K-1}{2}-2}, \sigma_{3}(Y), b_{\frac{K-1}{2}-1}, \tilde{\sigma}_{3}(R)\right),
\end{aligned}
$$

where $\sigma_{i}$ (resp. $\tilde{\sigma}_{i}$ ) puts the alternatives in $Y$ (resp. $R$ ) in some single-peaked order. As it can be checked, this profile $\succeq$ is single-peaked on $(X, H) .{ }^{18}$ In majority voting alternative $a$ defeats any alternative except $d$. $b_{1}$ defeats $d$ and, finally, $d$ defeats $a$.

\section{References}

Ballester M, Haeringer G (2011) A characterization of the single-peaked domain. Soc Choice Welf 36(2):305-322

Bandelt H-J (1985) Networks with Condorcet solutions. Eur J Oper Res 20(3):314-326

Bandelt H-J, Barthélémy J-P (1984) Medians in median graphs. Discret Appl Math 8(2):131-142

Black D (1948) On the rationale of group decision-making. J Polit Econ 56(1):23-34

Congleton R (2002) The median voter model. In: Rowley F, Schneider RK (eds) The Encyclopedia of public choice. Kluwer Academic Publishers, Dordrecht

Demange G (1982) Single-peaked orders on a tree. Math Soc Sci 3(4):389-396

Demange G (2011) Majority relation and median representative ordering. J Span Econ Assoc, 1-15

Downs A (1957) An economic theory of democracy. Harper and Row, New York

Eaton BC, Lipsey RG (1975) The principle of minimum differentiation reconsidered: some new developments in the theory of spatial competition. Rev Econ Stud 42(1):27-49

Eiselt HA, Laporte G (1989) Competitive spatial models. Eur J Oper Res, 39-231

Eiselt HA, Laporte G (1993) The existence of equilibria in the 3-facility Hotelling model in a tree. Transp Sci 27(1):39-43

Hansen P, Thisse JF, Wendell RE (1986) Equivalence of solutions to network location problems. Math Oper Res 11:672-678

Hotelling H (1929) Stability in competition. Econ J 39(153):41-57

Moulin H (1980) On strategy-proofness and single peakedness. Public Choice 35(4):437-455

Moulin H (1984) Generalized Condorcet-winners for single peaked and single-plateau preferences. Soc Choice Welf 1:127-147. doi:10.1007/BF00452885

\footnotetext{
17 Since $\mathcal{K}$ is a smallest circle, this is the only type of additional paths that are shortest paths between two members of the circle.

18 Note that a voter (e.g. voter 1) whose preference peak (alternative $a$ ) has several shortest paths to the opposite member of the circle (alternative $d$ ), must prefer all nodes on this path (a subset of $Y$ ) over the opposite member of the circle (alternative $d$ ). This is, however, the only restriction on the preference ranking between alternatives in $Y$ and alternatives on the circle for such a voter.
} 
Nehring K, Puppe C (2007a) Efficient and strategy-proof voting rules: a characterization. Games Econ Behav 59(1):132-153

Nehring K, Puppe C (2007b) The structure of strategy-proof social choice. Part I: general characterization and possibility results on median spaces. J Econ Theory 135(1):269-305

Osborne MJ, Slivinski A (1996) A model of political competition with citizen-candidates. Q J Econ 111(1):65-96

Roemer JE (2001) Political competition: theory and applications. Harvard University Press, Cambridge

Van de Vel M (1993) Theory of convex structures. North-Holland

Wendell RE, McKelvey RD (1981) New perspectives in competitive location theory. Eur J Oper Res 6(2):174-182 